\section{Allergie-Impfung mit nur vier Injektionen}

D ie WHO empfiehlt - neben der Karenz - die SIT mit Allergenen als einzige Behandlungsmethode, die den Verlauf einer Allergie positiv beeinflussen kann", erinnerte Th. Fuchs, Göttingen, an das 1998 veröffentlichte Positionspapier. Fuchs empfiehlt die SIT generell bei einer Beteiligung der Bronchien, einer Krankheitsdauer über vier Wochen sowie bei ungenügender Effizienz symptomatisch wirkender Medikamente. „Denken Sie daran, dass Antihistaminika keine Allheilmittel sind“, so der Vorsitzende des ÄDA.

Wichtig für die immunologische Wirkung der spezifischen Immunthera-

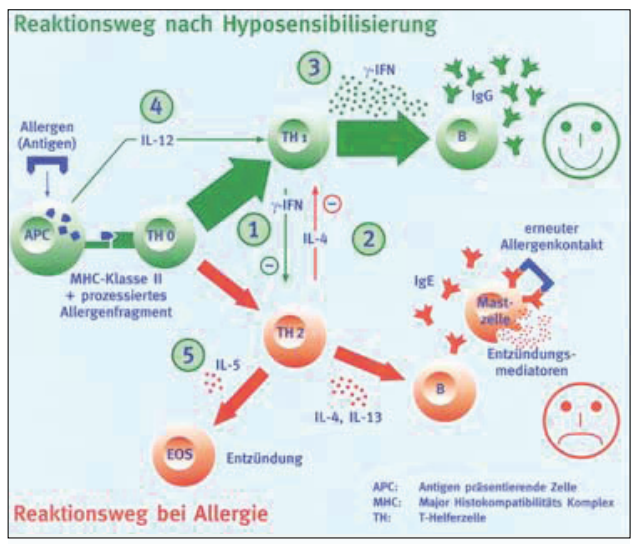

Schema der immunologischen Abläufe nach Hyposensibilisierung

pie sei vor allem eine „Richtungsänderung" von TH2- zu TH1-Zellantwort, erläuterte H.-J. Glowania, Koblenz (Abbildung). L. Klimek und B. Stuck untersuchten in einer plazebokontrollierten Studie die unter einer SIT zu erwartenden Änderungen der Zytokin-Produktion (Allergy 2001; 56: 93): Von 14 Baumpollen-Allergikern erhielten neun extrasaisonal vier SIT-Injektionen einer Baumpollen-Allergoid+Tyrosin+MPL ${ }^{{ }_{-}}$ Suspension, bei fünf Patienten injizierte man eine 2\%ige Tyrosin-Lösung. Die Mengen der geprüften Zytokine wurden vor SIT, nach SIT, intra- und postsaisonal bestimmt. Das Ergebnis: das aus den TH2-Zellen freigesetzte IL-4 zeigte in der Plazebogruppe an den letzten beiden Messzeitpunkten einen Anstieg; in der Verumgruppe hingegen fiel die IL-4-
Menge kontinuierlich ab und blieb signifikant unter den Werten der Plazebogruppe. Die Menge des gleichfalls aus den TH2-Zellen freigesetzten IL-5 war an den letzten beiden Messzeitpunkten bei den therapierten Patienten, im Vergleich zur Plazebogruppe, signifikant geringer. „Diese Ergebnisse zeigen, dass sich unter einer SIT eine Re-Organisation der TH2- in eine TH1-Präferenz einstellt", so Glowania abschließend.

K. J. Drachenberg, München, stellte eine doppelblinde, plazebokontrollierte Studie zur spezifischen Immuntherapie bei Gräser-Roggenpollen-Allergikern mit saisonaler allergischer Rhinitis vor (Allergy 2001; 56: 498-505). Die teilnehmenden 14 Zentren untersuchten Wirksamkeit und Verträglichkeit von TA Gräserpollen, jeweils versetzt mit $50 \mu \mathrm{g}$ MPL $^{\circledR}$ (POLLINEX Quattro). Die Patienten in der Verumgruppe erhielten vier Injektionen in wöchentlichen Abständen mit 300, 800 und 2 × $2000 \mathrm{SU}+50 \mu \mathrm{g} \mathrm{MPL}{ }^{\circledR} /$ Injektion $(\mathrm{n}=81)$, die Plazebogruppe $(\mathrm{n}=$ 60) 2\%ige L-Tyrosin-Lösung.

Nach vier Injektionen hatte sich der kombinierte Symptom-/Medikationsscore gegenüber Plazebo signifikant verbessert $(p=0,013)$. Die behandelten Patienten wiesen weniger Beschwerden und einen geringeren Verbrauch an symptomatisch wirksamen Medikamenten auf.

\begin{abstract}
$\mathrm{m}$ Rahmen des XX. EAACI-Kongress wurde erstmals der mit 10.000 Euro dotierte „Allergopharma Award“ verliehen. Preisträger ist der 39-jährige Cezmi Ali Akdis vom Schweizer Institut für Allergie- und Asthmaforschung in Davos. Er beschreibt in seiner Arbeit (FASEB J 2000; 14: 1666-68) Interaktionen zwischen Zytokinen und Rezeptorproteinen, die beim Gesunden sowie auch unter der spezifischen Immuntherapie zur Toleranzinduktion der zellulären Immunabwehr beitragen. Bei Allergikern ist dieser natürliche Prozess gestört, und es kommt zur Überreaktion des Immunsystems. Eine Schlüsselrolle - so die
\end{abstract}

\section{Adjuvanzien zur SIT}

Aluminiumsalz: Das modifizierte Allergen/Allergoid wird an Aluminiumsalze adsorbiert. Inzwischen ist dieses Adjuvans in die wissenschaftliche Diskussion geraten, da man - in Tierversuchen - feststellte, dass Aluminiumsalze potente $\mathrm{TH}_{2}$-Induktoren sind.

L-Tyrosin: Die Kombination von Allergen/Allergoid plus L-Tyrosin ist primär in Richtung TH1-Stimulation ausgerichtet. Der Vorteil gegenüber Aluminium-Adsorbaten liegt vor allem in der guten Verträglichkeit und der vollständigen Metabolisierung.

MPL ${ }^{\circledR}$ : Das Adjuvans Monophosphoryl-Lipid A (MPL $\left.{ }^{\circledR}\right)$ wird aus Zellwand-Lipopolysacchariden einer Subspezies von Salmonella minnesota gewonnen. Es verstärkt die Wirkung der Allergoide auf mehreren Ebenen der Immunantwort:

— Förderung der $\mathrm{TH}_{1}$-Produktion durch optimierte Allergen-Präsentation

- $\mathrm{TH}_{1}$-Zellen werden durch erhöhte Freisetzung von IL12 stimuliert und reagieren mit erhöhter Interferon- $\gamma$ und IL2-Produktion. Diese vermitteln einen Anstieg von IgG sowie eine Reduktion von IgE.

Fortbildung „Aktuelles aus Praxis und Forschung der Allergologie“, Hamburg 2001. Veranstalter: Bencard

\title{
Ausgezeichnetes zur Toleranzinduktion
}

Kernaussage Akdis - bei der T-Zell-Toleranzentwicklung spielt Interleukin 10.

Das auszeichenende Unternehmen Allergopharma engagiert sich besonders in der allergologischen Grundlagenforschung. Der Award wird von nun an jährlich für herausragende Arbeiten aus den Gebieten der allergischen Entzündungsreaktion sowie der Immuntherapie verliehen. Über die Homepage des Unternehmens www.allergopharma.de können Informationen zum Allergopharma Award 2002, Neapel, abgerufen werden.

Nach Informationen von Allergopharma, Reinbek 